\title{
PHYSICAL PARAMETERS FOR A CLASS OF HIGH-SPIN WAVE EQUATIONS
}

\author{
(Presented by H. Keres)
}

Relativistic wave equations connected with the representation $(A, A-1 / 2) \oplus$ $\oplus(A-1, A-1 / 2) \oplus(A-1 / 2, A-1) \oplus(A-1 / 2, A)$, are treated. The domain of free parameters which gives the physical mass spectrum is found. It is shown that for a one given spin value the masses may be chosen arbitrarily, then the other masses are uniquely determined by this choice. It turns out that the parameters corresponding to the Rarita-Schwinger equation lie in unphysical region.

1. In this paper we deal with the relativistic wave equations $\left(p_{\mu} \beta^{\mu}-m\right) \psi=0$, where $\psi$ transforms according to the representation $R(A)=(A, A-1 / 2) \oplus(A-1, A-1 / 2) \oplus(A-1 / 2, A-1) \oplus(A-1 / 2, A)$ and $A=1,3 / 2,2, \ldots$.

Depending on the choice of free parameters in $\beta^{0}$ matrix, one can vary the mass spectrum and get different types of equations with the same representation $R(A)$. Due to the acausality of high-spin oneparticle equations $\left[{ }^{1,2}\right]$, it becomes important to investigate different types of equations: single-mass, multi-mass, and barnacled equations. In this paper the domain of free parameters for $R(A)$ which gives the physical mass spectrum is found. The corresponding values of parameters are called the physical parameters.

The mass spectrum for $R(A)$ on certain restrictions on parameters was qualitatively studied in $\left[{ }^{3}\right]$, single-mass equations for $R(1)$ were found in $\left[{ }^{4-6}\right]$. In this paper we deal with the general case.

2. At first we deal with the representation $R(1)$ for spins $3 / 2$ and $1 / 2$. Masses are determined by the nonzero eigenvalues of $\beta^{0}$ matrix $\pm \lambda$ as follows: $m_{\lambda}=m / \lambda$. Physical masses correspond to real $\lambda$.

Denoting $1=(1,1 / 2), 2=(0,1 / 2), 3=(1 / 2,0)$ and $4=(1 / 2,1)$ we can represent $\beta^{0}=\beta^{(3 / 2)}+\beta^{(1 / 2)}$ in a general form $\left[{ }^{4-6}\right]$

$$
\beta^{(1 / 2)}=\left|\begin{array}{cccc}
0 & 0 & 0 & t_{14}^{3 / 2} \\
0 & 0 & 0 & 0 \\
0 & 0 & 0 & 0 \\
t_{41}^{3 / 2} & 0 & 0 & 0
\end{array}\right|,
$$


where $t_{i j}^{s}$ are spin-projection operators, $a, b$ and $c$ are free parameters which determined the masses of $s=1 / 2$ particles. For $\beta^{(3 / 2)}$ the nonzero eigenvalues are chosen to be \pm 1 , which means that the mass of $s=3 / 2$ particle is equal to $m$.

The investigation of nonzero eigenvalues of $\beta^{(1 / 2)}$ reduces to the eigenvalue problem of $4 \times 4$ matrix $\left[{ }^{6}\right]$

$$
\beta=\left|\begin{array}{cccc}
0 & 0 & a & 1 / 2 \\
0 & 0 & c & b \\
b & c & 0 & 0 \\
1 / 2 & a & 0 & 0
\end{array}\right| .
$$

The characteristical polynomial of $\beta$ is the following:

$$
\lambda^{4}-\left(2 a b+c^{2}+\frac{1}{4}\right) \lambda^{2}+\left(a b-\frac{c}{2}\right)^{2}=0
$$

From (4) we get

$$
2 \lambda_{1,2}^{2}=2 a b+c^{2}+\frac{1}{4} \pm\left(c+\frac{1}{2}\right) \sqrt{\left(c-\frac{1}{2}\right)^{2}+4 a b} .
$$

The eigenvalues of $\beta$ depend on two parameters - $a b$ and $c$. For physical mass values $\lambda_{1}^{2}$ and $\lambda_{2}^{2}$ must be real and nonnegative.

The physical values of parameters $a b$ and $c$ are suitable to represent on $a b-c$ diagram (Figure). The physical region is determined by the parabola

$$
4 a b=-\left(c-\frac{1}{2}\right)^{2}
$$

The points on the parabola correspond to the coincident eigenvalues $4 \lambda_{1}^{2}=4 \lambda_{2}^{2}=(c+1 / 2)^{2}$. These are also unphysical because the minimal polynomial of $\beta$ is $\left(\beta^{2}-\lambda_{1}^{2}\right)^{2}=0$. As it is anew demonstrated in $\left[{ }^{7}\right]$, such minimal polynomial gives the vanishing charge density and is therefore unphysical. On the parabola, there is the special point $(a b=-1 / 4, c=$ $=-1 / 2)$, where $\lambda_{1}=\lambda_{2}=0$ and $\beta$ is nilpotent. This special value of parameters is used when we want to describe single $s=3 / 2$ particle (then $\beta^{(1 / 2)}$ must be nilpotent). The causality considerations that will be given later allowed to regard the special point on the parabola as unphysical.

In physical region there are two notable lines: $c=-1 / 2$ and $a b=c / 2$ (Figure). On the line $c=-1 / 2$ there are points where $\lambda_{1}^{2}=\lambda_{2}^{2}=a b+1 / 4$ and $\beta$ satisfies $\beta^{2}-\lambda_{1}^{2}=0$. The corresponding points described two $s=1 / 2$ particles with the same mass $m / \lambda_{1}$. On the line $a b=c / 2$ there are points where $\lambda_{1}^{2}=(c+1 / 2)^{2}, \lambda_{2}=0$ and $\beta$ satisfies $\beta\left(\beta^{2}-\lambda_{1}^{2}\right)=0$.

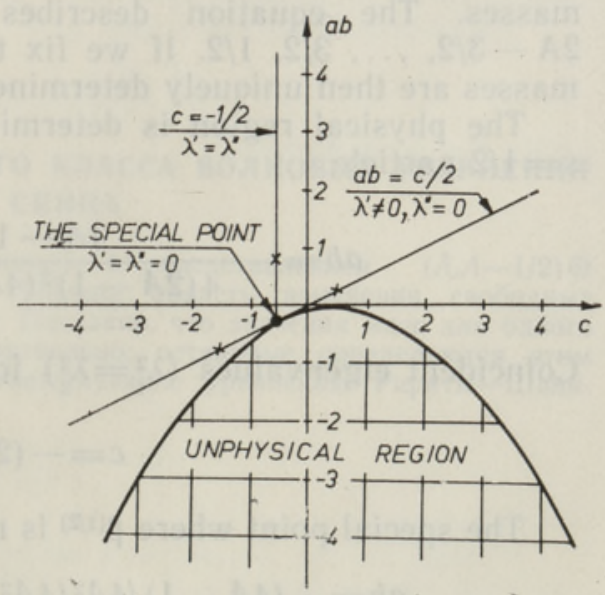


The corresponding points described single $s=1 / 2$ particle with mass $m / \lambda_{1}$.

The remainder points in the physical region described two $s=1 / 2$ particles with different masses. It is possible to verify that on tangents of the parabola (6), one of the eigenvalues is constant and the other varies from 0 to $\infty$. Therefore the masses of two $s=1 / 2$ particles may be chosen arbitrarily. For nonzero $\lambda_{1}$ and $\lambda_{2}\left(\lambda_{1} \neq \lambda_{2}\right)$, there are four different points in $a b-c$ diagram and therefore four different equations with the same mass spectrum. These equations have different parity combinations.

In previous papers $\left[{ }^{5,6}\right]$ we dealt with the single-mass equations for $R(1)$. These correspond to the following values of parameters: 1) $a b=$ $=-1 / 4, c=-1 / 2$ (the special point in Figure), corresponding to the Pauli-Fierz $s=3 / 2$ equation which, in turn, is equivalent to the RaritaSchwinger equation; 2) $a b=3 / 4, c=-1 / 2$, which corresponds to the Dirac equation for vector-bispinor and describes one $s=3 / 2$ and two $s=1 / 2$ particles; 3) $a b=1 / 4, c=1 / 2$ and $a b=-3 / 4, c=-3 / 2$, the corresponding two equations described one $s=3 / 2$ and one $s=1 / 2$ particle. The last three equations are denoted by crossed points in Figure.

In $\left[{ }^{3}\right]$ the qualitative mass, spectrum and parities for $R(A)$ were investigated, assuming that $\beta^{0}$ is hermitian. In this case we have an additional restriction $b=a^{*}$ and it limits for $a b$ the values where $a b \geqslant 0$. This restriction is superfluous because for $R(A)$ there always exists hermitizing matrix $\left[{ }^{6}\right]$ and therefore $\beta^{0}$ may be nonhermitian.

3. As we have mentioned above, in the free particle case also the special point on the unphysical parabola where $\beta$ is nilpotent, is used. It describes the single $\mathrm{s}=3 / 2$ particle (Pauli-Fierz, Rarita-Schwinger equation). In $\left[{ }^{1}\right]$ it has been shown that when the Rarita-Schwinger $s=3 / 2$ field is coupled minimally to an external electromagnetic field, the equation becomes acausal. From the results of $\left[{ }^{8,9}\right]$, it follows that all equations for which $\beta^{0}$ is diagonalizable, are causal in the presence of external electromagnetic field. Therefore all the equations with parameters in the physical region are causal because $\beta^{0}$ is diagonalizable.

Due to the fact that the only acausal equation corresponds to the special point in $a b-c$ diagram (Figure), which lies on the unphysical parabola; this special point must be regarded as unphysical. Therefore the equations with nilpotent matrices $\beta^{(8)}$ are also unphysical (we do not take into consideration nilpotency due to barnacles $\left.\left[{ }^{10}\right]\right)$.

4. In the case of general representation $R(A)$ there is also dependence on two parameters - $a b$ and $c$. Now we have less freedom to vary the masses. The equation describes particles with spins $s=2 A-1 / 2$, $2 \mathrm{~A}-3 / 2, \ldots, 3 / 2,1 / 2$. If we fix the masses for some spin $s$, the other masses are then uniquely determined.

The physical region is determined by the parabola corresponding to $s=1 / 2$ particle

$$
a b=-\frac{4 A-1}{4(2 A-1)^{2}\left(4 A^{2}-1\right)}\left(c-\frac{2 A-1}{2 A}\right)^{2} .
$$

Coincident eigenvalues $\left(\lambda_{1}^{2}=\lambda_{2}^{2}\right)$ for all spins lie on the line

$$
c=-(2 A-1) / 2 A \text {. }
$$

The special point where $\beta^{(1 / 2)}$ is nilpotent is

$$
a b=-(4 A-1) / 4 A^{2}\left(4 A^{2}-1\right), \quad c=-(2 A-1) / 2 A .
$$


The points where the other $\beta^{(s)}$ are nilpotent, lie in unphysical region. Therefore for $A>1$ there are no single-particle equations for $s=2 A-1 / 2$.

The lines where one of the eigenvalues is equal to zero, are different. It means that only for one spin value it is possible to choose $\lambda_{2}=0$, the other eigenvalues are nonzero.

In the $a b-c$ diagram there exists a point where for all spins the nonzero eigenvalues of $\beta^{(s)}$ are \pm 1 . It corresponds to parameters

$$
a b=-(4 A-1) / 4 A^{2}, \quad c=-(2 A-1) / 2 A .
$$

Now $\beta^{0}$ satisfies $\left(\beta^{0}\right)^{2}=1$, and the equation we get is equivalent to the Dirac equation for symmetrical traceless tensor-bispinor $\psi^{\mu_{1} \ldots \mu_{2 A-1}}$.

\section{REFERENCES}

1. Velo, G., Z w a nz i g e r, D., Phys. Rev., 186, № 5, 1337-1341 (1969).

2. Shamaly, A., Capri, A. Z., Ann. Phys. (N. Y.), 74, № 2, $503-523$ (1972).

3. B i r itz, H., Phys. Rev., D11, № 10, 2862-2869 (1975).

4. Lo id e, R.-K., Preprint FAI-10, Tartu, 1972.

5. Л ой де Р.-К., Изв. АН ЭССР, Физ. Матем., 23, № 3, 203-209 (1974).

6. Lo id e, K., Loide, R.-K., Preprint F-6, Tartu, 1977.

7. Cox, W., J. Phys. A: Math. Gen., 10, № 1, 109-113 (1977).

8. Am a r, V., D ozzio, U., Lett. Nuovo Cim., 12, № 17, 659-662 (1975).

9. Cox, W., J. Phys. A: Math. Gen., 9, № 4, 659-667 (1976),

10. Khalil, M. A. K., Progr. Theor. Phys., 58, № 5, 1538-1554 (1977). Academy of Sciences of the Estonian SSR,
Institute of Physics

Received Oct. 28, 1981

Tallinn Polytechnic Institute

M. KOIV, R.-K. LOIDE, R. SAAR

\section{UHE KORGEMA SPINNIGA VORRANDITE KLASSI FUUSIKALISED PARAMEETRID}

Artikkel käsitleb esitusega $(A, A-1 / 2) \oplus(A-1, A-1 / 2) \oplus(A-1 / 2, A-1) \oplus$ $\oplus(A-1 / 2, A)$ seotud relativistlikke lainevōrrandeid. On leitud vabade parameetrite muutumispiirkond, mis annab füüsikalise massispektri. On näidatud, et ühele fikseeritud spinnile võib massid valida suvaliselt, ülejäänud massid on aga selle valikuga määratud. On tõestatud, et Rarita-Schwingeri võrrandile vastavad parameetrid asetsevad mittefüüsikalises piirkonnas.

М. КЫИВ, Р.-К. ЛОИДЕ, Р. СААР

\section{ФИЗИЧЕСКИЕ ПАРАМЕТРЫ ДЛЯ ОДНОГО КЛАССА ВОЛНОВЫХ УРАВНЕНИИ ВЫСШЕГО СПИНА}

Рассмотрены волновые уравнения, связанные с представлением $(A, A-1 / 2) \oplus$ $\oplus(A-1, A-1 / 2) \oplus(A-1 / 2, A-1) \oplus(A-1 / 2, A)$. Найдена область изменения свободных параметров, даюих физнческий спектр масс. Показано, что значения масс для одного фиксированного спина можно выбрать произвольно, остальные определяются этим выбором. Установлено, что параметры, соответствующие уравнению Рариты-Швингера, лежат в нефнзической области. 